\title{
Process Model Consistency Measurement
}

\author{
Sukanth Sistla \\ CSE Department, JNTUniversity, Anantapur, Andhra Pradesh, India.
}

\begin{abstract}
In software development, managers increasingly focus on process improvement which in turn has increased the demand for software metrics. To turn the business application of a software project to system specification is a big challenge in business environment. Since business analysts and system analysts have their own perspective, the modeling of business process is necessary to facilitate both the perspectives and for a better coordination. An attempt is made to resolve inconsistencies and to develop model that verifies process consistency described in Petri net graph. From this model it has been confirmed that the consistency ranges from 0.0 to 1.0. In addition to the above Support Vector Machines (SVM) usage is helpful to improve consistency with greater confidence to evaluate behavioral and structural consistency.
\end{abstract}

Keywords: behavioral profiles, behavioral equivalence, change propagation, consistency checking, process model.

\section{Introduction}

To eliminate the gap [2][9] between business applications and system specifications, business analysts and system analysts have their own perspective needed to be coordinated properly, many applications of such business processing model[1] have given raise to problems and maintaining consistency of such related models has become a challenge for business modeling practice. Behavioral profile is a solution to the inappropriateness of behavioral notions and also change propagation between models including inconsistencies can be resolved. Through this model free-choice Petri nets [4] with reference to their places and transitions, profiles can be computed. Schema integration [5] in particular schema matching investigates and shows such correspondences can be identified automatically. Methodologies for integrated system design like matching techniques and graphical matching can also be applied. Targeting research challenge of defining a notion of consistency between process models [3] is more adequate than existing notions of behavioral equivalence. Behavioral profiles are less sensitive to projections than trace equivalence of as behavioral profiles remain unchanged even if start and end branches are introduced. Profile consistency [1][3] ranging from 0 to 1.0. The proposed change uses Support vector machines can to improve consistency with greater confidence.

\section{Consistency Measurement Using Behavioral Profiles And Structural Analysis}

Business process change [10] is at the very core of business process management, reaching from business evolution to process enactment, results in multiple models that overlap in content due to serving different purposes. That, in turn, imposes serious challenges for the propagation of changes between these process models.

\subsection{Process Models}

Our notion of a process model [3][8] is based on a graph containing activity nodes and control nodes, which, in turn, captures the commonalities of process description languages. Thus, the subset of BPMN used in our initial example can be traced back to the following definition of a process model.

Definition 1: (Process Model) A process model [10] is a tuple

$\mathrm{P}(\mathrm{A}, \mathrm{I}, \mathrm{C}, \mathrm{F}, \mathrm{T})$ with

$\mathrm{A}$ as a non-empty set of activity nodes, and $\mathrm{C}$ as a set of control nodes, $\mathrm{A}$ and $\mathrm{C}$ are disjoint,

I as a set of initial activities,

$\mathrm{F}$ as the flow relation, and

$\mathrm{T}$ : $\{$ and, or, $\mathrm{xor}\}$ as a function that assigns a type to control nodes.

\subsection{Behavioral Profile}

The Behavioral profile [1] aims at capturing Behavioral aspects of a process in a fine-grained manner. That is, it consists of three relations between nodes of a process graph. These relations are based on the notion of weak order [3]. Thus, weak order does not have to hold for all traces of the model.

○ The strict order relation $x \succ P(y)$ and $y \neg \succ P(x)$

$\circ \quad$ The exclusiveness relation $x \neg \succ P(y)$ and $y \neg \succ P(x)$ 
- The observation concurrency relation $x \succ P(y)$ and / or $y \succ P(x)$

The set of all three [8] relations is the Behavioral profile.

Two process models with equivalent behavioral profiles may differ in the trace equivalence, in contrast the two process models with identical trace equivalence can also identical in behavioral profiles.

Correspondence Relation: if the relation between two process models is left unique and is not functional Projected Firing sequence: In a sequence considered, the set of aligned sequences is referred as firing sequence. Trace Consistency of Alignment: If Aligned transitions of a projected firing sequence contain trace equivalence then it reflects as Trace consistency of alignment.

\subsection{Structural Analysis}

The structural analysis of dynamic lumped process models [8] forms an important step in the model building procedure and it is used for the determination of the solvability properties of the model, too. This analysis includes the determination of the degree of freedom, structural solvability, differential index and the dynamic degrees of freedom. As a result of the analysis, the decomposition of the model is obtained and the calculation path can be determined. This way the appropriate numerical method for solving the model can be chosen efficiently. Moreover, advice on how to improve the computational properties of the model by modifying its form or its specification can also be given.

Effective graph-theoretical methods have been proposed in the literature based on the analysis tools developed by, for the determination of the most important solvability property of lumped dynamic models: the differential index. The properties of the dynamic representation graph of process models described by semiexplicit DAE-systems have also been analyzed there in case of index 1 and higher index models. Beside the algorithm of determining the differential index by using the representation graph, a model modification method has also been proposed in the literature, which results in a structurally solvable model even in the case of higher index models.

\subsection{Dynamic Representation Graph}

A dynamic graph is a sequence of static graphs corresponding to each time step of the integration. On a dynamic graph there are directed arcs attached from the previous static graph to the succeeding static graph that are determined by the method applied for solving the ordinary differential equations. In case of a single step explicit method, the value of a differential variable at time $\boldsymbol{t} \boldsymbol{t} \boldsymbol{h}$ is computed using the corresponding differential value and its value at a previous tune $t$. For example, when the explicit Euler method is used:

$$
x(t+h)=x(t)+h \cdot x^{\prime}(t) \rightarrow e q(1)
$$

Where $\boldsymbol{h}$ denotes the step length during the numerical integration. The structure of a dynamic graph assuming explicit Euler method for solving differential equations is shown in figure 1.

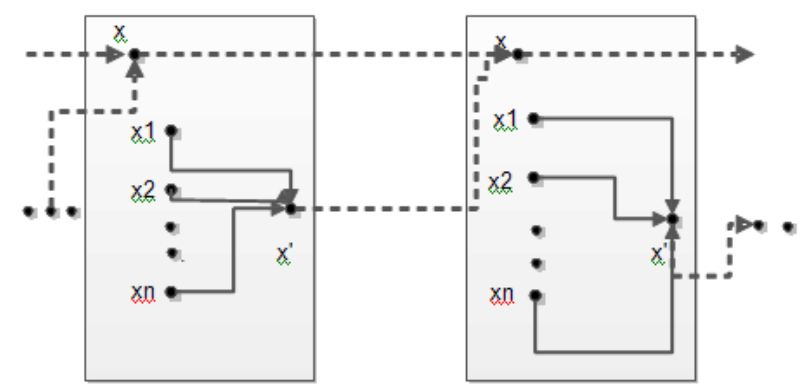

Fig.1 Dynamic Graph model for Euler method for solving differential

The structural analysis based on graph theoretical technique is carried out in steps performed sequentially. The first step is to rewrite the model into its standard form. The second step is the assignment of types to vertices in the representation graph. The important types of vertices determined by the model specification are the following:

- $\quad\langle S\rangle($ set)-type variables: These represent variables, which are assigned to the specified given values. In the case of a dynamic representation graph assuming explicit method for solving the differential equations, the differential variables will be labelled by type $\left\langle S^{*}\right\rangle$ because their initial value can be 
obtained from the initial values, and then their values can be calculated step by step by numerical integration. Labels $\langle S\rangle$ and $\left\langle S^{*}\right\rangle$ are treated the same way during the analysis.

- $\langle\mathrm{G}\rangle$ (given)-type variables: A variable assigned to a specific value of a left hand side is a $\langle\mathrm{G}\rangle$-type variable. Unlike the $\langle\mathrm{S}\rangle$-type variables, the values of the right hand side variables will be suitably adjusted so as to preserve the equality of the two sides.

\section{Consistency Measures For Aligned Process Models}

The previously defined concept of a behavioral profile [1][3] allows us to formally discuss the notion of a degree of profile consistency between a pair of process models.

\subsection{Consistency based on Behavioral Profiles}

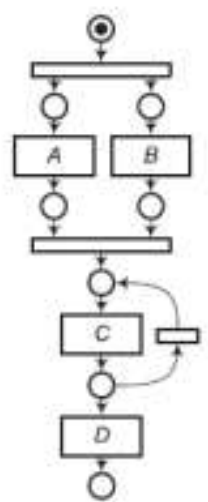

(a)

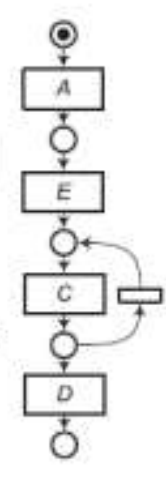

(b)

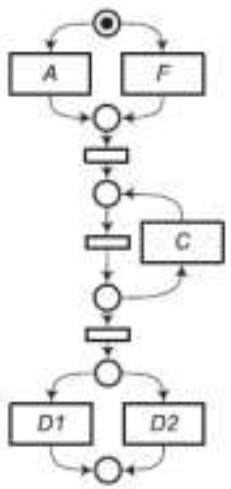

(c)

Fig.2 Exemplary alignment setting.

In general, our notion of consistency based on behavioral profiles, i.e., profile consistency, is grounded on the preservation of behavioral relations for corresponding activities. In contrast to the notion of a trace consistent alignment, it does not require the correspondence relation to be injective. Instead, it allows for 1:n (and even $\mathrm{n}: \mathrm{m}$ ) correspondences. Therefore, this notion can be applied to vertical as well as horizontal alignments. Preservation of the behavioral relation is only required in case there are no overlapping correspondences. With respect to the examples in Figure 2. it is easy to see that all pairs of aligned transitions are also consistent with respect to their behavioral relation. For instance, the strict order relation between transitions A and D in model 2(a) is preserved for transition pair A and D1, as well as A and D2 in model 2(c). In addition, in all three models it holds $\mathrm{C} \| \mathrm{C}$. That is, $\mathrm{C}$ might occur multiple times during execution.

\subsection{Interpretation of Profile Consistency}

As exemplified in the previous section, the degree of profile consistency ranges between 0 and 1.0 for two process models and a correspondence relation. Still, a degree of 1.0 does not imply that both models are (projected) trace equivalent. This stems from the fact that the underlying behavioral profile represents a behavioral abstraction; apparently, the degree of profile consistency quantifies the quality of an alignment with respect to the order of potential activity occurrences. A degree of 1.0 guarantee the all these constraints are equal for the aligned activities of two models. A degree of 0.9, in turn, indicates that the constraints on the order of potential activity occurrences are equal solely for $90 \%$ of the relations between aligned activities. As the degree of profile consistency measures the quality of the alignment, its definition is independent of the coverage of the process models by the correspondence relation (i.e., the share of activities in both models that are aligned). Based on the degree of profile consistency, consistency thresholds might be defined.

However, we assume these thresholds to be highly dependent on a specific project setting. Once a degree of profile consistency below 1.0 is observed, the question of how to locate the source of inconsistency has to be addressed. According to our approach, inconsistencies manifest themselves in different relations of the behavioral profile of two process models for a pair of aligned activities. This information can directly be provided to business analysts and system analysts in order to judge on the necessity of the inconsistency. While this kind of feedback allows for locating the inconsistency directly in case of only a few inconsistent profile relations (e.g., caused by an interchanged order of two activities in a sequence), it might be inappropriate if a big number of profile relations is inconsistent. 


\section{Experiments And Results Analysis}

After preprocessing of the benchmark models, we are able to analyze its consistency. As mentioned before, we establish correspondences between events and functions with equal labels. Further on, we extract all pairs of process models that are aligned by at least two correspondences. For such a pair, we then calculate the consistency measures, that is, trace consistency, the degree of trace consistency, and the degree of profile consistency of the alignment and finally analyzed the accuracy of the degree of profile using structural analysis. The results are optimistic from the experiments conducted on bench mark business models represented in Petrinet format. We consider the consistency measurement systems WF systems (WF) [6], and Behavior profiling (BP) analysis to compare with the proposed Behavior Profiling and Structural Analysis (BP\&SA) [8]. We can find the significant benefit of BP\&SA over other models [7]. Figure 2 represents the comparison of optimality in consistency measurement between BP\&SA and other two models [7]. In figure 3 we can observe the computational over head of the WF [6]. Here BP is having slight advantage over BP\&SA, which can be negligible while considering the accuracy achieved through BP\&SA in consistency measurement.

TABLE 1 Overall Results

\begin{tabular}{|l|l|l|l|}
\hline Technique & Precision & Recall & F-Score \\
\hline $\begin{array}{l}\text { Lexical N- } \\
\text { M without } \\
\text { stemming }\end{array}$ & 0.72 & 0.60 & 0.68 \\
\hline $\begin{array}{l}\text { Lexical N- } \\
\text { M with } \\
\text { stemming }\end{array}$ & 0.72 & 0.60 & 0.66 \\
\hline $\begin{array}{l}\text { A-Star } \\
\text { with Post } \\
\text { Processing }\end{array}$ & 0.81 & 0.60 & 0.69 \\
\hline Greedy & 0.89 & 0.60 & 0.72 \\
\hline
\end{tabular}

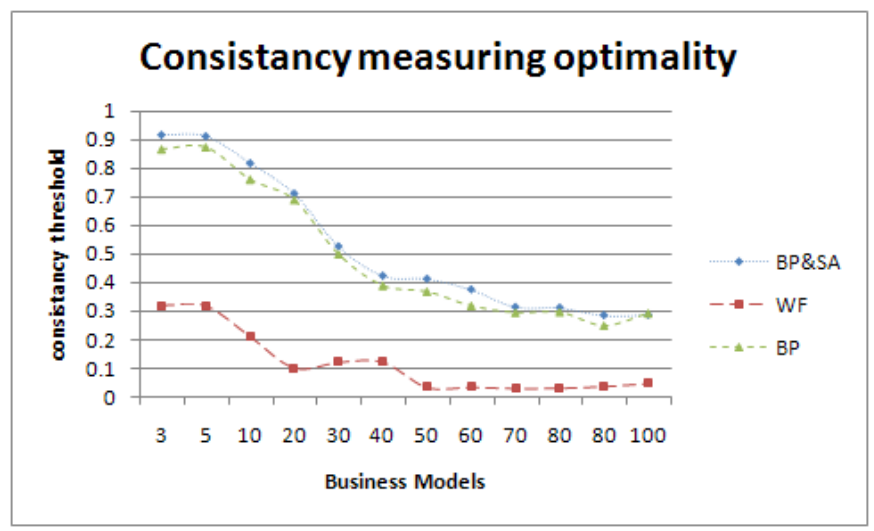

Fig.3 Optimality in Consistency Measurement

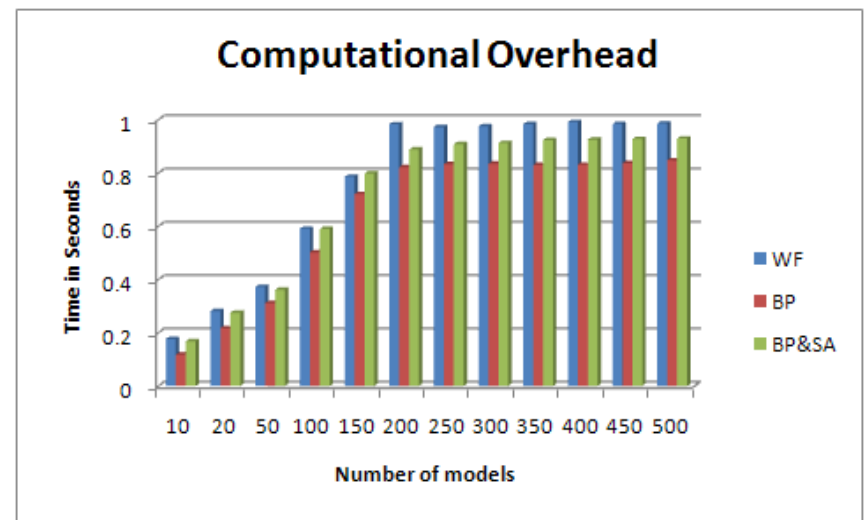

Fig.4 Computational Overhead comparison report 


\section{Conclusions}

Process models play an important role to reduce the gap between business requirements and system specifications. In this article, we have discussed addressed the research challenge of defining a notion of consistency between process models that is more adequate to this problem than existing notions of behavioral equivalence. Behavioral profiles are used for the definition of the formal notion of profile consistency. Behavioral profiles provide three major advantages in contrast to the existing notion of trace equivalence and consistency measures that build up it. First, behavioral profiles are less sensitive as behavioral profiles remain unchanged even if additional start and end branches are added. Second, the structure of a behavioral profile provides degree of profile consistency ranging from 0 to 1.0 and Structural analysis accurate the consistency measurement through degree of profile. Finally, the concept of a behavioral profile builds informal properties of free-choice Petri nets. We proved that profile consistency can be checked for sound free-choice WF-systems in $\mathrm{O}(\mathrm{n} 3)$ time with n nodes. and to evaluate behavioral and structural consistency SVM is helpful.

\section{Journal Papers:}

\section{References}

[1] M. Weidlich, M. Weske and J. Mendling, "Efficient Consistency Measurement Basedon Behavioral Profiles of Process Models", IEEE Trans. Software Eng.,vol. 37, no. 3, pp. 410-429, May 2011.

[2] C. Rolland and N. Prakash, "Bridging the Gap between Organisational Needs and ERP Functionality," Requirements Eng., vol. 5, no. 3 , pp. $180-193$, Oct. 2000

[3] G. Rull, C. Farre and E. Teniente "Validation of Mappings between Schemas," Data and Knowledge Eng., vol. 66,no. 3, pp. 414437, 2008.

[4] W.M.P. van der Aalst, “The Application of Petri Nets to Workflow Management,"J.Circuits Systems and Computers, vol. 8, no. 1,pp. 21-66, 1998.

[5] C. Rolland and N. Prakash, "Bridging the Gap BetweenOrganisational Needs and ERP Functionality," RequirementsEngineering, vol. 5, no. 3, pp. 180-193, October 2000.

\section{Proceedings Papers:}

[6] M. Weidlich, M. Weske and J. Mendling, "Change Propagation in Process Models Using Behavioural Profiles,” Proc. IEEE Int'l Conf.Services Computing, 2009.

[7] M. Weidlich, R. Dijkman, and J. Mendling, "The ICoP Framework:Identification of Correspondences between Process Models," Proc.22nd Int'l Conf. Advanced Information Systems Eng., 2010.

[8] M. Weidlich, J. Mendling and Sergey Smirnov "Business Process Model Abstraction based on Behavioral Profiles", Proc. IEEE Int'l Conf. Services Computing, 2009.

\section{Chapters in Books:}

[9] N. Lohmann, E. Verbeek, and R.M. Dijkman, "Petri Net Transformations for Business Processes—A Survey," Trans. PetriNets and Other Models of Concurrency, vol. 2, pp. 46-63, Springer-Verlag, 2009.

[10] R. M. Dijkman, "Diagnosing Differences between Business Process Models," in BPM, ser. Lecture Notes in Computer Science, M. Dumas, M. Reichert, and M.-C. Shan, Eds., vol.5240. Springer, 2008, pp. 261-277. 